\title{
Escenarios barriales urbanos: espacios de producción de vulnerabilidades en la adolescencia y juventud en el Gran Buenos Aires, Argentina
}

\author{
Urban neighborhood scenarios: spaces for the production \\ of vulnerabilities in adolescence and youth in Greater Buenos Aires, \\ Argentina
}

\author{
Gabriela Wald y Alejandro Capriati \\ Instituto de Investigaciones Gino Germani, Universidad de Buenos Aires / \\ CONICET, Argentina
}

Resumen

En este trabajo presentamos los primeros resultados de un estudio cuali-cuantitativo en el cual se abordan las heterogeneidades existentes en las experiencias juveniles de desigualdad. La investigación analiza escenarios barriales específicos en los suburbios de la Ciudad de Buenos Aires, Argentina, y los vincula con las trayectorias biográficas de adolescentes y jóvenes que los habitan. En este trabajo se avanza, primero, en la conceptualización de la noción de escenario barrial, pues la misma permite captar las posiciones estructurales y diferencias de oportunidad constreñidas a un área determinada. Luego, se reconstruyen empíricamente dos escenarios barriales, los cuales dan cuenta de escenarios sociales típicos que pueden hallarse en los suburbios de la Ciudad de Buenos Aires y de otros conglomerados urbanos del país. Mediante este abordaje buscamos especificar disparidades territoriales e inequidades socio-espaciales en dos barrios seleccionados, a la vez que analizar de qué maneras las especificidades de un territorio influyen en la vida cotidiana y la salud de quienes viven en él.

Palabras clave: Juventud, desigualdad, vulnerabilidad, escenario social, barrios populares.

\section{Abstract}

In this paper we present the first results of a qualitative-quantitative study in which the existing heterogeneities in youth experiences of inequality are addressed. The research analyzes specific neighborhood scenarios in the suburbs of the City of Buenos Aires, Argentina, and links them with the biographical trajectories of adolescents and young people who inhabit them. This work advances, first, in the conceptualization of the notion of neighborhood scenario, since it allows us to capture the structural positions and differences of opportunity constrained to a given area. Then, two neighborhood scenarios are empirically reconstructed, which account for typical social scenarios that can be found in the suburbs of the City of Buenos Aires and other urban conglomerates in the country. Through this approach we seek to specify territorial disparities and socio-spatial inequalities in two selected neighborhoods, while analyzing in what ways the specificities of a territory influence the daily life and health of those who live in it.

Key words: Young people, inequalities, vulnerabilities, scenario, disadvantaged neighborhoods. 


\section{INTRODUCCIÓN}

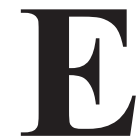

n Argentina, como en América Latina, gran parte de la población adolescente y joven crece y vive con derechos vulnerados: no concurren a la escuela ni logran finalizar el nivel secundario, trabajan en el mercado informal en condiciones precarias y, en particular, las mujeres realizan tareas domésticas y de cuidado en sus hogares sin reconocimiento ni remuneración. Las condiciones de vida y el acceso a recursos y oportunidades está atravesado por marcadas desigualdades que expresan patrones de segregación espacial, de clase, de género y pertenencia étnica.

$\mathrm{Al}$ abordar estos procesos sociales emerge con fuerza la necesidad de comprender más acerca de los modos en que las intersecciones de las desigualdades se expresan en la vida de las personas y los lugares que habitan. En las últimas dos décadas se han desarrollado relevantes aportes desde las ciencias sociales, en particular desde los campos de estudios sobre espacialidad, exclusión y juventud, para vislumbrar la dimensión espacial de las desigualdades sociales y la concentración territorial de desventajas (Saraví y Serrano Santos, 2020; Segura, 2020; Bayón, 2015; Kessler y Dimarco, 2013; Di Virgilio y Perelman, 2014; Steinberg et al., 2017).

Para contribuir con los avances realizados por estas áreas de estudio desde una perspectiva de salud y derechos humanos, hemos decidido abordar el estudio de las desigualdades sociales en adolescentes y jóvenes en su nivel territorial de máxima desagregación, así como los modos en que la población joven logra hacer frente a las mismas. ${ }^{1}$ La construcción de este objeto de estudio obedece a que aún no conocemos lo suficiente cómo operan los mecanismos mediante los cuales las desigualdades se superponen, qué factores influyen más o menos en estas superposiciones, en qué medida los recursos con los que cuentan las mujeres y varones jóvenes (familiares, institucionales, programáticos) son utilizados y pueden aliviar situaciones de vulnerabilidad, si determinadas cuestiones culturales - por ejemplo los repertorios morales en los cuales han sido socializadas las personas- afectan estos procesos, o en qué medida la política pública está colaborando con procesos de igualdad, entre otros interrogantes que se sitúan en las mediaciones entre la estructura y la agencia.

\footnotetext{
${ }^{1}$ Este artículo presenta la construcción del problema y los primeros resultados del estudio "Heterogeneidades en sectores populares. Escenarios de inclusión-exclusión y trayectorias biográficas en adolescentes y jóvenes de dos partidos del Gran Buenos Aires, Argentina”, Proyecto de Investigación Científica y Tecnológica (PICT) trianual periodo 2016-2019, financiado por el Ministerio de Ciencia y Técnica de Argentina. Investigadores responsables: Gabriela Wald y Alejandro Capriati.
} 
Hemos definido dos componentes para avanzar en esta línea de análisis. Por un lado, describimos y analizamos escenarios sociales específicos donde transcurren las vidas de adolescentes y jóvenes residentes en barrios populares del Gran Buenos Aires. Por el otro, y en línea con nuestras investigaciones previas (Wald, 2012; Capriati, 2014), analizamos las trayectorias biográficas de un conjunto de adolescentes y jóvenes, así como sus horizontes de expectativas ${ }^{2}$ para el futuro. Nuestro interés radica en analizar cómo se intersectan e influyen estos dos componentes entre sí. Para hacerlo, trabajamos con la complementariedad de perspectivas micro, meso y macro sociales. Nuestro enfoque se propone comprender cómo los procesos históricos se internalizan en las vidas de las personas (Bourgois, 2003) — vía instituciones y aspectos culturales mediadores - a la vez que dilucidar cómo y por qué las personas se ubican de un determinado modo frente a dichos procesos históricos.

En este artículo presentamos resultados del primer componente de nuestra investigación, realizada entre los años 2016 y 2019 en barrios populares de dos partidos del Gran Buenos Aires: en San Francisco y Santa Rosa, ${ }^{3}$ al sur del partido de Morón; y en Villa Fiorito, partido de Lomas de Zamora.

Presentamos el proceso metodológico mediante el cual reconstruimos dos tipos de escenarios barriales. Dicha reconstrucción la hemos realizado a partir de la definición de cinco dimensiones, las cuales dan cuenta de distintos aspectos de los barrios analizados: i) la infraestructura urbana; ii) las condiciones de las viviendas y los hogares; iii) los niveles educativos de adultos y población en edad escolar; iv) el tejido institucional local; y v) el clima social barrial. Hacia el final del artículo conceptualizamos cada escenario barrial y planteamos interrogantes sobre la vinculación de los mismos con las trayectorias biográficas de los adolescentes y jóvenes que viven en ellos.

\section{Estudios sobre desigualdades y vulnerabilidades}

Para enmarcar teóricamente la investigación hemos puesto en diálogo los aportes de los estudios sobre espacialidad, exclusión y juventudes con estudios sobre vulnerabilidades en salud y derechos humanos. Las desigual-

\footnotetext{
${ }^{2}$ Horizonte de expectativas es una categoría proveniente de la metodología del análisis biográfico. Alude a la esperanza, a la posibilidad o a lo deseable y también a la voluntad; remite a lo que cada persona espera para sí del porvenir, formando parte del calendario privado de cada sujeto (Kosellek, 1985). En algunos casos estos horizontes de expectativas pueden tomar la forma de proyectos biográficos - aunque no es posible afirmar que todas las personas logran articular este tipo de relato de aquello que esperan sobre su propia vida.

${ }^{3}$ Los barrios de Santa Rosa y San Francisco a los fines de la investigación constituyen una misma zona de campo.
} 
dades sociales y la segregación socioespacial forman parte constitutiva de la salud en sentido amplio, entendida en tanto aspectos colectivos e integrales que favorecen o limitan el bienestar de las poblaciones.

La segregación residencial es un fenómeno de larga data en las ciudades latinoamericanas (Saraví y Serrano Santos, 2020), pues las mismas se han expandido desde la década de 1970 con un patrón de fragmentación y urbanización excluyente (centros y periferias, zonas ricas y zonas pobres), como consecuencia de una política neoliberal que posibilitó el uso desregulado del suelo urbano (Segura, 2020).

El interés por el barrio y la dimensión local no nos hace perder de vista que las oportunidades o desventajas no remiten al barrio en sí mismo, sino a todo el territorio urbano. El barrio es un espacio que forma parte de una red de interdependencias espaciales más amplia, condicionada por la estructura urbana (Saraví y Serrano Santos, 2020; Di Virgilio y Perelman, 2014).

El espacio urbano, su producción y configuraciones constituyen una realidad material y relacional de producción y reproducción de desigualdades (Segura, 20204). La accesibilidad a la ciudad, la proximidad o los recursos con los que cuentan las poblaciones a nivel local son constitutivos de estos procesos.

Enmarcado en estos debates, proponemos para este trabajo retomar la noción de escenario social, pues la misma permite captar las posiciones estructurales y diferencias de oportunidad constreñidas a un área determinada (Steinberg et al., 2011). Al estudiar fenómenos vinculados con la población joven, particularmente cuestiones de su salud integral, los enfoques descontextualizados tienden a reforzar interpretaciones que atribuyen los problemas a la ignorancia de las personas afectadas, culpabilizándolas por no adherir a comportamientos de cuidado, exagerando su autonomía.

Retomamos asimismo el marco de análisis en vulnerabilidad y derechos humanos (Ayres, Paiva, Buchalla, 2018), entendiendo la vulnerabilidad como un conjunto de aspectos individuales y colectivos vinculados con una mayor susceptibilidad a padecer perjuicios y menor disponibilidad de recursos para su protección. Desde este enfoque se consideran tanto las prácticas de las personas como sus relaciones sociales, y el impacto del Estado, por acción u omisión (Pecheny, 2013).

\footnotetext{
${ }^{4}$ En la mayor parte de las perspectivas analíticas, los territorios son tratados como lugares no problemáticos sobre los cuales operan variables independientes (globalización, neoliberalismo) que impactan en variables dependientes (por ejemplo, el mercado de trabajo o la educación) (Segura, 2020)
} 
Finalmente, la condición juvenil en tanto objeto de estudio condensa un nodo atravesado por la edad en su intersección con el género, la clase social, la condición étnica, las culturas juveniles, entre otros clivajes de la vida social. ${ }^{5}$ En la experiencia de transición a la adultez, el espacio urbano, con sus ventajas o desventajas, aparece como un componente central (Saraví y Serrano Santos, 2020).

\section{ESTRATEGIA METODOLÓGICA}

La estrategia metodológica de nuestro estudio combina las siguientes técnicas cuantitativas y cualitativas para hacerlas dialogar:

1. La descripción de la estructura sociodemográfica de la población joven $\mathrm{y}$ algunos indicadores de condiciones de vida en cada uno de los barrios seleccionados a partir del análisis de datos censales (2010) procesados para este estudio por fracciones o radios censales correspondientes a cada uno de los territorios analizados. Estos datos se contextualizan con los de sus respectivos partidos, y algunos de ellos con datos sobre los 24 partidos del conurbano bonaerense.

2. La descripción de los recursos comunitarios y programáticos existentes en cada uno de los barrios a partir de un relevamiento propio realizado vía observación participante y entrevistas con referentes de organizaciones territoriales y actores estatales en las dos zonas de campo.

3. La identificación de situaciones de vulnerabilidad, así como de los soportes o apoyos de los que echan mano para hacerles frente, y de los repertorios morales que pregonan los/las adolescentes y jóvenes de ambos barrios mediante la utilización de la metodología del relato de vida. ${ }^{6}$

Esta estrategia metodológica combina la técnica del relato de vida -realización de una serie de tres o cuatro entrevistas a una misma persona- con el análisis del territorio donde estas personas viven, y el releva-

\footnotetext{
${ }^{5}$ La demarcación de un rango de edad de la adolescencia y juventud están moldeadas por la cultura y el contexto. En la actualidad, el período de transición de la infancia a la adultez ocupa cada vez más una mayor porción del curso de la vida (Sawyer, Azzopardi, Wickremarathne, Patton, 2018).

${ }^{6}$ En el relato de vida no se trata de explorar todos los sucesos de la vida, sino de comprender la trayectoria a partir de la identificación de los acontecimientos que la han determinado (Leclerc-Olive, 2009). Son relatos en los cuales, a través de varias entrevistas, se despliegan las experiencias de una persona y permiten acceder al testimonio subjetivo y a las valoraciones que hace de su propia vida (Di Leo y Camarotti, 2013; Pujadas Muñoz, 1992). El desafío que propone esta técnica radica en vincular la experiencia, única e individual de un sujeto, con el contexto social, para comprender los sentidos de la experiencia y los procesos sociales que en ella se desenvuelven (Kornblit, 2010).
} 
miento de los recursos institucionales y programáticos disponibles a nivel barrial.

Seleccionamos los barrios de San Francisco/Santa Rosa y Villa Fiorito porque dan cuenta de dos escenarios sociales típicos presentes en el conurbano bonaerense, los cuales presentan algunas similitudes a la vez que una serie de diferencias sustantivas que afectan las condiciones de vida y de salud de los y las adolescentes y jóvenes. Mediante este abordaje buscamos especificar disparidades territoriales e inequidades sociales en dos barrios seleccionados, a la vez que analizar de qué maneras las especificidades de cada territorio influyen en la vida cotidiana y la salud de los y las jóvenes que viven en ellos.

En este artículo presentamos resultados de los puntos uno y dos a partir de la noción de escenario barrial, la cual nos permite develar similitudes y diferencias entre las dos zonas de campo seleccionadas, a la vez que realizar una primera definición conceptual que alude a cuestiones tanto macro como meso sociales, de modo de articular teóricamente nuestra propuesta metodológica. Como ya hemos adelantado, definimos cinco dimensiones para la reconstrucción de dos escenarios barriales del conurbano bonaerense, algunas de ellas corresponden a aspectos de tipo estructural, otras son de tipo institucional y otras de índole representacional. Asimismo, se seleccionaron indicadores cuantitativos en base a información censal i) que esté disponible para ser procesada en su máximo nivel de desagregación - por fracciones o radios censales_-; ii) que, correspondiendo al año 2010, implique información de lenta modificación que pueda dar cuenta de las condiciones de vida de las poblaciones en la actualidad. ${ }^{7}$

Para la descripción de la estructura sociodemográfica y algunos datos de las condiciones de vida de los barrios se utilizó la herramienta de manejo de datos de gran volumen REDATAM, ${ }^{8}$ la cual permitió procesar información por radios censales (un conjunto de manzanas en el caso de los barrios de Morón) o fracciones censales (un conjunto de radios censales en el caso de Villa Fiorito). Si bien la información disponible corresponde al año

\footnotetext{
7 A modo de ejemplo, hemos seleccionado indicadores como "calidad constructiva de la vivienda" o "calidad de conexión a servicios básicos" en detrimento de otros como "cantidad de computadoras en el hogar" o "condición de ocupación" pues estos últimos, ya sea por el avance de las tecnologías de la información y la comunicación o por la amplia definición que abarca la categoría "ocupado" para el cuestionario del Censo Nacional de Población, Hogares y Vivienda, no nos resultaron lo suficientemente descriptivos de las condiciones de vida actuales en los barrios seleccionados.

${ }^{8}$ REDATAM es el acrónimo de REcuperación de DATos para Áreas pequeñas por Microcomputador, se trata de un sistema computacional que facilita el procesamiento, análisis y diseminación web de la información de censos, encuestas, registros administrativos, indicadores nacionales/ regionales y otras fuentes de datos. Ha sido desarrollado por el Centro Latinoamericano y Caribeño de Demografía (CELADE), División de Población de la CEPAL.
} 
2010, las variables estructurales (por ejemplo, si existe trazado cloacal activo) han sido cotejadas en los relevamientos barriales, con lo cual, sumado al hecho de que son aspectos de lenta modificación, aportan información aún válida desagregada localmente. Los datos a nivel local se comparan en todos los casos con los datos de sus respectivos partidos, los cuales permiten mostrar las brechas de desigualdad en las áreas bajo estudio.

Para el relevamiento de la infraestructura y la caracterización de los barrios se realizaron observaciones semanales en ambas zonas de campo, 18 entrevistas en profundidad a referentes de organizaciones y de instituciones gubernamentales en los barrios de Morón Sur, y 12 entrevistas en profundidad y un grupo focal en Villa Fiorito todo entre los meses de agosto y diciembre de 2016. La selección de los barrios obedeció al criterio de heterogeneidad para lograr una mayor potencia descriptiva.

\section{Dos escenarios del Conurbano bonaerense: Villa Fiorito (Lomas DE Zamora) y Santa Rosa y SAN Francisco(MorónSUR)}

En este apartado reconstruiremos empíricamente dos tipos de escenarios barriales que podemos hallar en el conurbano bonaerense. Por sus características y condiciones de vida, los barrios de Villa Fiorito y de Morón Sur nos hablan de muchos otros barrios de ese conurbano infinito, como han denominado Rodrigo Zaragaza y Lucas Ronconi (2017) al territorio de 24 partidos densamente poblado que rodea a la Ciudad Autónoma de Buenos Aires. En él viven más de 10 millones de personas, de las cuales 33 por ciento son adolescentes y jóvenes de entre 10 y 29 años de edad (INDEC, 2010).

Los partidos de Lomas de Zamora y Morón forman parte del primer cordón del Área Metropolitana de Buenos Aires, el primero al sur y el segundo al oeste de la misma. La zona de campo de Morón comprende 54 manzanas y su población alcanzaba en el año 2010 los 6,334 habitantes. La zona de campo de Lomas de Zamora comprende alrededor de 180 manzanas, y su población ascendía en 2010 a 27,831 habitantes. Es importante aclarar que se incluye en este estudio un área de asentamientos que se radicaron en la zona de Fiorito con posterioridad al censo, luego del entubado del Arroyo Unamuno y el posterior relleno de terrenos con basura. Sobre esas poblaciones, que se estiman en 2,500/3,000 personas, no hay disponible información censal, la información censal que presentamos excluye entonces a estos hogares. La zona de campo de Villa Fiorito es la más 
densamente poblada: posee $12,775 \mathrm{hab} / \mathrm{km}^{2}$ frente a los $9,756 \mathrm{hab} / \mathrm{km}^{2}$ de Santa Rosa y San Francisco. ${ }^{9}$

La población que tiene entre 10 y 29 años corresponde a 37 por ciento en San Francisco y Santa Rosa, y a 39.5 por ciento en la zona de campo de Villa Fiorito, superando tanto el porcentaje de los 24 partidos del conurbano bonaerense como el de sus respectivos partidos.

\section{Infraestructura urbana}

Hemos reconstruido esta dimensión a partir de observaciones y entrevistas a informantes clave, la cual se completa con información del censo 2010 procesada para estos barrios.

Villa Fiorito difiere de Morón y Santa Rosa en su morfología, en las fuentes de contaminación con las cuales conviven sus poblaciones y en los servicios básicos disponibles.

En San Francisco y Santa Rosa el trazado barrial es en forma de damero, las calles poseen nombre y las viviendas están numeradas. La mayor parte del territorio está asfaltado (alrededor de 70 por ciento), la provisión de luz por medidores está extendida (aunque no cubre todo el territorio), la recolección de basura se realiza de manera habitual y existen zonas a las que llega el gas natural de red. La red cloacal está en construcción desde mediados de la década del 2010, aunque aún no en funcionamiento - según los habitantes del barrio sólo falta una conexión, pero la misma estaría demorando años. Existe también una fuente de contaminación: una usina asfáltica ubicada en los límites del barrio. Sobre ella hay controversias y vecinos organizados; no obstante, la misma continúa en funcionamiento. En estos barrios no hay asentamientos ni zonas pobladas sin planificación. La mayoría de las familias son dueñas de los terrenos sobre los que han construido sus viviendas, así es que la mayor parte de las familias cuenta con un espacio propio para vivir, y por lo tanto, estabilidad habitacional.

Villa Fiorito, por su parte, se conformó tras una sucesión de asentamientos que fueron organizándose y regularizándose a lo largo del tiempo. La zona de campo seleccionada refleja esta heterogeneidad, y presenta distintos grados de urbanización. Comprende un área de casas bajas, generalmente de material, con trazado en forma de damero, calles pavimentadas y veredas transitables. El acceso a servicios básicos es heterogéneo en esta área: unas pocas cuadras tienen cloacas y acceso al agua de red, mientras

${ }^{9}$ La densidad de población ha sido calculada combinando datos censales con la herramienta de cálculo de superficie de google maps. Para ello fue necesaria la georeferenciación de los radios y fracciones censales con un software especializado, pues dicha georeferenciación no estaba disponible en Redatam. 
que la situación generalizada está signada por la falta de acceso a agua segura y los déficits en el sistema de desagües cloacales y pluviales. En estas zonas la estabilidad habitacional es similar a la que hallamos en Santa Rosa y San Francisco. La zona de los asentamientos tiene una organización irregular, con viviendas precarias, pequeñas y de chapa, sin acceso a agua de red ni desagües cloacales, sin acceso a pozos sépticos. En esta zona, gran parte de las viviendas no tiene baños en su interior; es común que los mismos estén afuera, y que algunos baños como canillas sean de uso comunitario. La tenencia de las tierras es irregular, pues se sostiene en la ocupación, y hay tensiones políticas y sociales respecto a los asentamientos.

En Villa Fiorito los factores contaminantes son múltiples y refieren a la problemática ambiental de la cuenca Matanza-Riachuelo. ${ }^{10}$ Como consecuencia de ello una parte de la población padece contaminación por metales pesados, especialmente por plomo. La zona también está afectada por la proliferación y quema de basura - quema, que en los asentamientos, al no haber recolección de basura, es diaria. Finalmente, este cuadro de situación se ve potenciado por las inundaciones: al subir las napas contaminadas, dichas inundaciones representan un serio riesgo para la salud de estas poblaciones.

El INDEC presenta tres categorías para definir la calidad de servicios básicos de un territorio, las cuales refieren a la tenencia de agua y a los desagües: "calidad satisfactoria" (viviendas que disponen de agua potable de la red pública y desagüe cloacal), "calidad básica" (viviendas que disponen de agua de red pública y desagüe a pozo con cámara séptica) y "calidad insuficiente" (viviendas que no cumplen ninguna de las dos condiciones anteriores).

Los datos para los barrios seleccionados se muestran en la Los datos muestran profundas inequidades entre los partidos y las zonas de campo, a la vez que una mayor brecha en Morón: mientras que el partido de Morón en su conjunto tiene 54 por ciento de hogares con calidad satisfactoria de conexión a servicios básicos (frente a 33 por ciento de Lomas de Zamora), en Morón Sur 100 por ciento de los hogares posee una insuficiente calidad de conexión a servicios básicos (pues no hay red cloacal trazada en el barrio y tampoco agua de red en de los hogares).

${ }^{10}$ La Cuenca Matanza Riachuelo es un curso de agua de $64 \mathrm{~km}$ que nace en la Provincia de Buenos Aires, Argentina. Integra la lista de los cauces más contaminados del mundo, como consecuencia de la actividad industrial circundante, del desagote de cloacas y de los residuos sólidos arrojados en ella. A su alrededor viven alrededor de seis millones de personas (15 por ciento de la población del país). En la zona se producen inundaciones con frecuencia a causa de las precipitaciones y la pendiente natural del río, que se suman a la falta de impermeabilidad de los suelos y a la ausencia de infraestructura que permita el drenaje del agua. 
Tabla 1: Calidad de conexión a servicios básicos por zonas de campo y partidos

\begin{tabular}{lcccc}
\hline & Morón & $\begin{array}{c}\text { San Francisco } \\
\text { y Sta. Rosa }\end{array}$ & $\begin{array}{c}\text { Lomas de } \\
\text { Zamora }\end{array}$ & $\begin{array}{c}\text { Villa } \\
\text { Fiorito }\end{array}$ \\
\hline Satisfactoria & $54 \%$ & $0 \%$ & $33 \%$ & $8 \%$ \\
Básica & $20 \%$ & $0 \%$ & $30 \%$ & $30 \%$ \\
Insuficiente & $26 \%$ & $100 \%$ & $37 \%$ & $62 \%$ \\
\hline
\end{tabular}

Fuente: elaboración propia, basados en el Censo Nacional de Hogares, Población y Viviendas, 2010 (INDEC).

En Villa Fiorito, si bien la gran mayoría de las viviendas censadas posee acceso a agua segura, 62 por ciento de los hogares aún se agrupa dentro de la categoría de insuficiente dada la baja cantidad de viviendas conectadas a desagües cloacales. Recordemos que estos datos no incluyen los asentamientos no censados, en los cuales es habitual la provisión de agua a través de canillas comunitarias que están siempre excedidas en demanda.

Tenemos entonces dos escenarios barriales con infraestructura urbana deficiente, siendo Morón Sur de características más homogéneas en cuanto a su trazado y organización y Villa Fiorito más heterogéneo, con presencia de asentamientos donde la infraestructura urbana es inexistente y con un alto grado de contaminación ambiental.

\section{Condiciones de las viviendas y los hogares}

La segunda dimensión de análisis de los escenarios barriales aborda aspectos estructurales sobre las viviendas, así como la presencia de marcadores de pobreza estructural como las necesidades básicas insatisfechas (NBI).

El índice "calidad constructiva de la vivienda", de acuerdo a la elaboración del INDEC, toma en cuenta la calidad de los materiales de la vivienda, adónde tienen la canilla más cercana y cómo es el baño. El mismo tiene tres categorías: i) calidad satisfactoria: refiere a las viviendas que disponen de materiales resistentes, sólidos y con la aislación adecuada. A su vez también disponen de cañerías dentro de la vivienda y de inodoro con descarga de agua; ii) calidad básica: si bien cuentan con cañerías dentro de la vivienda y de inodoro con descarga como el anterior, no cuentan con elementos adecuados de aislación o tienen techo de chapa o fibrocemento; iii) calidad insuficiente: engloba a las viviendas que no cumplen ninguna de las dos condiciones anteriores. 
Los porcentajes de viviendas dentro de cada categoría en nuestras zonas de campo y partidos se muestran en la Tabla 2.

Tabla 2: Calidad constructiva de la vivienda por zonas de campo y partidos

\begin{tabular}{lcccc}
\hline & Morón & $\begin{array}{c}\text { San Francisco } \\
\text { y Sta. Rosa }\end{array}$ & $\begin{array}{c}\text { Lomas de } \\
\text { Zamora }\end{array}$ & $\begin{array}{c}\text { Villa } \\
\text { Fiorito }\end{array}$ \\
\hline Satisfactoria & $77 \%$ & $38 \%$ & $62 \%$ & $32 \%$ \\
Básica & $18 \%$ & $33 \%$ & $21 \%$ & $32 \%$ \\
Insuficiente & $5 \%$ & $29 \%$ & $17 \%$ & $36 \%$ \\
\hline
\end{tabular}

Fuente: elaboración propia, basados en el Censo Nacional de Hogares, Población y Viviendas, 2010 (INDEC).

La Tabla 2 muestra que, en San Francisco, Santa Rosa y Villa Fiorito la mayor parte de las viviendas presentan calidad de construcción básica o insuficiente - mostrando también mayores inequidades entre los barrios de Morón Sur y los totales del partido. En el mismo sentido se expresa la cantidad de hogares con al menos una Necesidad Básica Insatisfecha (NBI), ${ }^{11}$ tal como se muestra en la Tabla 3.

Tabla 3: Al menos un indicador de NBI

\begin{tabular}{lcccc}
\hline & Morón & $\begin{array}{c}\text { San Francisco y } \\
\text { Sta. Rosa }\end{array}$ & $\begin{array}{c}\text { Lomas de } \\
\text { Zamora }\end{array}$ & $\begin{array}{c}\text { Villa } \\
\text { Fiorito }\end{array}$ \\
\hline Hogares sin NBI & $96 \%$ & $88 \%$ & $91 \%$ & $90 \%$ \\
Hogares con NBI & $4 \%$ & $12 \%$ & $9 \%$ & $10 \%$ \\
\hline
\end{tabular}

Fuente: elaboración propia, basados en el Censo Nacional de Hogares, Población y Viviendas, 2010 (INDEC).

De la Tabla 3 se desprende que la existencia de al menos una NBI en los barrios bajo estudio es cercana al promedio del conurbano bonaerense, que es de 9.2 por ciento (INDEC, 2014). Los datos procesados para esta segunda dimensión muestran que los hogares y viviendas de los barrios bajo estudio se parecen más entre sí que con sus respectivos partidos, con condiciones habitacionales deficientes en ambos, y con cerca de diez por

11 El INDEC considera hogares con NBI a aquellos en los cuales está presente al menos uno de los siguientes indicadores de privación: NBI 1: Hogares que habitan viviendas con más de tres personas por cuarto (hacinamiento crítico). NBI 2: Hogares que habitan en una vivienda de tipo inconveniente (pieza de inquilinato, hotel, pensión, vivienda precaria o vivienda no destinada a tal fin). NBI 3: Hogares que habitan en viviendas que no tienen retrete o tienen retrete sin descarga de agua. NBI 4: Hogares que tienen algún niño en edad escolar (6 a 12 años) que no asiste a la escuela. NBI 5: Hogares que tienen cuatro o más personas por miembro ocupado y en los cuales el jefe tiene bajo nivel de educación (no completó tercer grado del nivel primario) INDEC (2014). 
ciento de hogares con NBI. Exhiben también que existe una mayor inequidad al interior del partido de Morón, donde Santa Rosa y San Francisco presentan una brecha mayor de desigualdad con el total del partido que la que hallamos en Villa Fiorito - cuyo partido, Lomas de Zamora, presenta a su vez peores condiciones de vida que el de Morón.

\section{Asistencia escolar y niveles educativos}

La tercera dimensión de caracterización de escenarios barriales hace alusión a la educación. En primer lugar, decidimos procesar el nivel educativo de los jefes y jefas de hogares, dada la fuerte relación entre este indicador y la situación de pobreza y desigualdad de niños y jóvenes (UNICEF, 2017).

La Tabla 4 muestra que la mayor parte de los jefes y jefas de hogar de las zonas de campo poseen la primaria como máximo nivel de estudio alcanzado, cosa que no ocurre en los partidos de los que forman parte. En los barrios estudiados, entonces, hay una enorme mayoría de jefes y jefas que nunca asistió al colegio secundario (59 por ciento en Morón Sur y 68 por ciento en la zona de campo de Villa Fiorito).

Tabla 4: Nivel educativo de jefes/as de hogar por zona de campo y partidos

\begin{tabular}{lcccc}
\hline & Morón & $\begin{array}{c}\text { San Francisco } \\
\text { y Sta. Rosa }\end{array}$ & $\begin{array}{c}\text { Lomas de } \\
\text { Zamora }\end{array}$ & $\begin{array}{c}\text { Villa } \\
\text { Fiorito }\end{array}$ \\
\hline Primario incompleto & $8 \%$ & $19 \%$ & $14 \%$ & $27 \%$ \\
Primario completo & $24 \%$ & $40 \%$ & $31 \%$ & $41 \%$ \\
Secundario incompleto & $16 \%$ & $22 \%$ & $18 \%$ & $14 \%$ \\
$\begin{array}{l}\text { Secundario completo } \\
\text { Estudios terciarios }\end{array}$ & $24 \%$ & $14 \%$ & $25 \%$ & $15 \%$ \\
$\begin{array}{l}\text { o de grado incompletos } \\
\begin{array}{l}\text { Estudios terciarios } \\
\text { o de grado completos }\end{array}\end{array}$ & $12 \%$ & $3 \%$ & $6 \%$ & $2 \%$ \\
\hline
\end{tabular}

Fuente: elaboración propia, basados en el Censo Nacional de Hogares, Población y Viviendas, 2010 (INDEC).

La segunda variable educativa procesada fue la población en edad escolar que asiste a la escuela (Tabla 5). 
Tabla 5: Asistencia a la escuela de niños/as y adolescentes por partido según edad

\begin{tabular}{lcccccccc}
\hline & \multicolumn{2}{c}{ Morón } & \multicolumn{2}{c}{$\begin{array}{c}\text { San Francisco } \\
\text { y Sta. Rosa }\end{array}$} & \multicolumn{2}{c}{$\begin{array}{c}\text { Lomas de } \\
\text { Zamora }\end{array}$} & \multicolumn{2}{c}{$\begin{array}{c}\text { Villa } \\
\text { Fiorito }\end{array}$} \\
& Asiste & No asiste & Asiste & No asiste & Asiste & No asiste & Asiste & No asiste \\
\hline 5-14 años & $99 \%$ & $1 \%$ & $99 \%$ & $1 \%$ & $98 \%$ & $2 \%$ & $96 \%$ & $4 \%$ \\
$15-19$ años & $88 \%$ & $12 \%$ & $73 \%$ & $27 \%$ & $72 \%$ & $28 \%$ & $61 \%$ & $39 \%$ \\
\hline
\end{tabular}

Fuente: elaboración propia, basados en el Censo Nacional de Hogares, Población y Viviendas, 2010 (INDEC).

Observamos que la asistencia a la escuela primaria y a los primeros años de la escuela secundaria está ampliamente extendida tanto en los partidos como en las zonas de campo, mientras la asistencia a los últimos años de la escuela secundaria decrece en todas las zonas analizadas, siendo mayor la brecha de abandono escolar en los barrios bajo estudio que en sus respectivos partidos. Si bien en San Francisco y Santa Rosa casi un tercio de los adolescentes no asisten a la escuela —o no lo hacían en el año 2010 - Villa Fiorito muestra los indicadores más preocupantes con casi 40 por ciento de jóvenes por fuera del colegio secundario.

A partir del análisis combinado de indicadores de educación de la población adulta y joven podemos diferenciar los niveles educativos: mientras en Morón sur el nivel educativo es de características medio-bajas (aun cuando estos indicadores muestran grandes déficits educativos), la zona de campo de Villa Fiorito presenta un nivel educativo bajo, con valores aún más deficientes que los de Morón sur.

\section{El tejido institucional barrial}

La cuarta dimensión mediante la cual reconstruimos los escenarios barriales bajo estudio refiere a las organizaciones e instituciones que funcionan en cada uno de ellos, así como a los programas locales disponibles y los modos de acceso por parte de las poblaciones. Es una dimensión que aborda aspectos meso sociales de las desigualdades y sus modos de enfrentarlas en los barrios analizados, al analizar la oferta institucional local de manera comparativa. Hemos decidido incluir esta dimensión por la centralidad que tienen las organizaciones e instituciones para la gestión de la vida cotidiana de las comunidades que viven en estos barrios. Las mismas vehiculizan muchas veces el acceso a recursos del Estado (becas, cursos de formación, bolsones de alimentos, etcétera), funcionan como espacios de encuentro 
o de esparcimiento, reflejan en ocasiones necesidades de las poblaciones locales y facilitan el cumplimiento de derechos.

A partir de observaciones y entrevistas a informantes clave es posible afirmar que en ambas zonas de campo existen organizaciones tanto del Estado como comunitarias que cubren, con mayor o menor nivel de déficit según el área y el barrio, las áreas de educación (jardines, primarias, secundarias, secundarias para adultos y escuelas privadas parroquiales), salud (centros de APS), deporte y cultura. Ambos barrios cuentan también con espacios religiosos (católicos y evangélicos), de militancia política, comedores y merenderos.

Asimismo, en zonas próximas a los barrios (no más de 15 cuadras) registramos la existencia de servicios de protección de derechos de niños, niñas y adolescentes, de atención de los consumos problemáticos de drogas (en su mayoría de espacios religiosos), así como servicios municipales descentralizados. En ninguna de las zonas de campo existen escuelas para la población con discapacidad y tampoco universidades. Para acceder a estas últimas es preciso realizar un trayecto de media hora en transporte público en ambas zonas de campo.

No obstante, estas similitudes iniciales, el grado de organización de las instituciones difiere de manera sustancial en las dos zonas que analizamos, y lo mismo ocurre con las instituciones que trabajan con población joven.

En Morón Sur existen pocas organizaciones de la comunidad, los adultos sólo participan en organizaciones partidarias o en un centro de jubilados que gestiona una referente comunitaria. El débil tejido institucional tiene su correlato en la escasa oferta para la población joven, donde sólo una ONG, Antilquito, que nos acompaña en el trabajo de campo, está profesionalizada. Hay otras dos instituciones que nuclean adolescentes y jóvenes, una de ellas municipal (La casa Joven, algo alejada del barrio) y la sociedad de Fomento, que sólo ofrece fútbol para varones. El territorio tiene presencia elevada de iglesias y de espacios de militancia política.

En relación al vínculo entre instituciones, relevamos que existió un intento de construir una red entre los barrios vecinos, pero al momento del trabajo de campo, si bien la red aún existía, no había proyectos en común.

En Villa Fiorito, por el contrario, el entramado institucional es denso, con algunas organizaciones grandes de larga trayectoria en la zona. Por ejemplo, el Foro Hídrico de Lomas de Zamora nuclea organizaciones y vecinos que demandan sus derechos ambientales y de acceso al agua segura y desagües cloacales. En los asentamientos los vecinos se organizan a través de referentes comunitarios elegidos por las familias. 
El MTE (Movimiento de Trabajadores Excluidos) es la mayor organización en el territorio, de la cual participa una parte significativa de la población cartonera. En numerosas ocasiones la población se ha organizado ante necesidades puntuales por fuera de espacios políticos específicos. Vale mencionar también que existe trabajo en red, con reuniones periódicas (generalmente mensuales) en las cuales participan la Delegación $\mathrm{Mu}-$ nicipal, Escuelas, Centros de Salud, ONG y otros actores del barrio para abordar cuestiones comunes.

Específicamente en el trabajo con jóvenes, se destaca la Fundación Che Pibe, con 30 años de trabajo comunitario en Fiorito y protagonista junto a otras organizaciones del Colectivo por los Derechos de la Infancia y Adolescencia. Che Pibe es la institución que nos acompaña en nuestro trabajo de campo en esa zona, una de las más antiguas y profesionalizadas. Participa en diversas redes de trabajo con organizaciones locales, provinciales, organizaciones de la cultura e impulsa reclamos barriales que surgen en la comunidad - reducción del plomo en sangre, acceso al agua potable - problematizando las demandas de las familias que viven en los asentamientos.

En Villa Fiorito, además, está el Centro Cultural "Floreal Ferrara", que ofrece actividades para jóvenes y mujeres de prevención contra la violencia de género y el consumo problemático de sustancias, además de otros talleres culturales. También hay organizaciones como la Autoridad de Cuenca Matanza-Riachuelo (ACUMAR) vinculadas a las problemáticas ambientales.

Se observa que los espacios comunitarios en Villa Fiorito son de mayor envergadura, funcionan en terrenos y edificios propios, y están más profesionalizados que los de Morón Sur. La mayoría tienen gran cantidad de años de trabajo en el territorio, con un grado de institucionalización mayor.

Lo expuesto hasta aquí nos permite afirmar que el entramado institucional en las dos zonas de campo bajo estudio presenta diferencias significativas. Tenemos entonces, por un lado, un escenario barrial en Morón sur con un entramado institucional escaso, poco profesionalizado, de baja incidencia en la población y con poco trabajo en red. En Villa Fiorito, por el contrario, se observa un entramado institucional denso, profesionalizado, politizado, en contacto con los actores relevantes del territorio (estatales y no estatales), con alta participación de la población y con gran trayectoria de trabajo en red - ya sea para demandar derechos al Estado como para la organización de actividades barriales. 


\section{El clima social en los barrios}

El concepto de clima social barrial ha sido utilizado para describir variables que van desde las tasas de crímenes y violencias hasta el apoyo social existente, el tejido institucional, la estabilidad en la posesión de una vivienda, la cohesión vecinal y la eficacia de la acción colectiva (Sellström y Bremberg, 2006). En el campo de la educación y la promoción de la salud, se ha propuesto la noción de clima social escolar (Kornblit, 2008), definido como "las percepciones que tienen los sujetos acerca de las relaciones interpersonales que establecen en el contexto escolar y el contexto o marco en el cual estas interacciones se dan" (Kornblit, 2008: 59).

En el marco de nuestra investigación, definimos la noción de clima social barrial como las percepciones acerca de la desprotección que manifiestan las y los residentes de las zonas de campo sobre su seguridad física y material, ya sea en relación con los robos y crímenes, los enfrentamientos entre bandas, la violencia institucional, como así también a las percepciones de protección que perciben las comunidades por parte de sus instituciones o redes barriales. Entendemos que este agregado de percepciones de carácter intersubjetivo enlazan aspectos objetivos y subjetivos; de modo similar a la definición de sensación de inseguridad como entramado de discursos y emociones, juicios morales y temores (Kessler, 2009).

Esta dimensión de los escenarios barriales ha sido reconstruida a partir de entrevistas a informantes clave y de las observaciones y visitas realizadas por nuestro equipo durante el año 2016 en cada una de las zonas de campo. Es una dimensión que ubicamos a la vez en el meso y el micronivel de análisis, pues vincula aspectos subjetivos con situaciones experimentadas por los distintos grupos que viven en un territorio dado.

En Villa Fiorito y en los barrios del sur de Morón el clima social difiere de manera sustancial.

En las conversaciones mantenidas a lo largo del relevamiento comunitario en Villa Fiorito, en escuelas y centros de salud, en organizaciones sociales y delegaciones municipales, aparecen referencias permanentes a la exposición a distintas violencias, más o menos organizadas, y a la sensación de desprotección frente al crimen. La violencia policial y/o la convivencia de las fuerzas policiales con el delito, los robos en la calle o en la entrada y salida del hogar, son referencias frecuentes en los discursos de las y los referentes comunitarios.

Acompañando estos discursos, pudimos constatar que en los meses en los cuales se realizó la primera etapa del relevamiento comunitario (agosto - diciembre 2016), Villa Fiorito fue noticia en distintos medios de comu- 
nicación y redes sociales por cuestiones policiales y de justicia: causas por trata de personas, allanamientos por tráfico de drogas y corrupción policial.

Así como priman las percepciones de violencia y desprotección en las descripciones que realizan los informantes clave y entrevistados/as sobre el clima social de Villa Fiorito, los referentes de organizaciones y algunos de los jóvenes también reconocen que la acción colectiva en el barrio puede generar acciones de protección - $\mathrm{O}$ al menos la demanda por lo que reconocen como sus derechos a una vida digna libre de violencia institucional y estigmatización de los jóvenes.

En San Francisco y Santa Rosa, por su parte, las menciones a la violencia y al crimen aparecieron poco en los relatos de los informantes clave entrevistados. Si bien sí se mencionaron "los chicos de la esquina" y asociado a ellos el consumo de drogas (sin especificar cuáles) y las peleas entre bandas de jóvenes (entre estos barrios, pero sobre todo con barrios vecinos), las referencias a la existencia de narcotráfico, crimen organizado y a la violencia en las calles fueron escasas. No se mencionaron dificultades para circular por el barrio, aunque si referencias a sentirse más seguros cuando hay "gente en la calle", o que en el horario de la siesta "hay más chances de que te agarren". Sí aparecieron en algunos relatos de vida escenas de enfrentamientos entre jóvenes del barrio con barrios, vecinos pero haciendo referencia al pasado, manifestando que en el presente el barrio estaba tranquilo, los "pibes que hacían kilombo" están en la cárcel o "se calmaron", y no se manifiesta la existencia de "mulos, transas y todo eso". Estos discursos se contextualizan con la nula aparición de San Francisco y Santa Rosa en los medios de comunicación, en las noticias policiales y en las redes sociales.

Finalmente, hubo un aspecto en el que sí hallamos grandes diferencias y fue en el estigma vinculado al territorio que se habita. En Villa Fiorito diversos jóvenes y referentes institucionales mencionaron que a veces es mejor "no decir que vivís en Fiorito" por la carga negativa que tiene para el mundo "de afuera". Referentes comunitarios relataron que aconsejan a los jóvenes que buscan trabajo mencionar algún barrio vecino. Nada de esto encontramos en las entrevistas y conversaciones informales con adolescentes y adultos de los barrios de Morón Sur.

Tenemos entonces un escenario barrial, Villa Fiorito, cuyo clima social podríamos calificarlo como de elevada violencia, cuyos habitantes manifiestan temor al caminar por las calles, describen la existencia de redes de crimen organizado, vínculos caldeados entre distintos grupos de la población y con la policía, cuyas instituciones pueden funcionar en conjunto 
para ofrecer algún tipo de protección. Por el otro, tenemos un escenario barrial con clima social de violencia baja en Morón sur, con escasas referencias al temor de circular por el barrio, a enfrentamientos violentos entre barrios, a robos, así como nulas referencias a la presencia de grupos de crimen organizado.

Los estigmas asociados al territorio son un mecanismo de regulación y control de la sociabilidad (Serrano, 2019) y constituyen una fuente de vulnerabilidad con consecuencias en la autoestima y emociones de adolescentes y jóvenes (Saraví y Serrano Santos, 2020).

\section{ESCENARIOS BARRIALES QUE LIMITAN EL EJERCICIO DE LA SALUD Y LOS DERECHOS}

Hemos desarrollado hasta aquí las cinco dimensiones seleccionadas para caracterizar los escenarios sociales de los barrios donde realizamos nuestro estudio. Los datos nos permiten observar similitudes y diferencias, evidenciando cierta heterogeneidad dentro de lo que podemos agrupar como barrios populares. ${ }^{12}$

Tenemos entonces un primer escenario barrial, que observamos en San Francisco y Santa Rosa (Morón Sur), en el cual la infraestructura urbana es deficiente, aunque homogénea, las condiciones de vivienda son de nivel bajo, los niveles educativos de la población son medio-bajos, el tejido institucional es débil con poca experiencia de trabajo en red y el clima social es descrito como de baja violencia. En segundo lugar, tenemos el escenario barrial de Villa Fiorito cuya infraestructura urbana es deficiente, donde hay asentamientos con infraestructura urbana nula y con alta contaminación ambiental, las condiciones de vivienda son deficientes, los niveles educativos son bajos, el tejido institucional es tupido y con trayectoria de trabajo en red y el clima social en las calles es descrito como de muy alta violencia.

Así, el análisis de las cinco dimensiones abordadas nos permite definir la existencia de dos tipos de escenarios barriales populares, a partir de la utilización de indicadores macro y meso sociales, por medio de los cuales se identifican diferencias en las posiciones estructurales y en las oportunidades vinculadas a cada área.

Estas inequidades socio espaciales superpuestas que hemos documentado en este trabajo - tanto entre Villa Fiorito y Morón Sur como entre estos barrios y sus respectivos partidos - son productoras de vulnerabilidades.

12 Entendemos por barrio popular a villas de emergencia, asentamientos o barrios de trazado urbano donde habitan mayormente personas de niveles socioeconómicos medio-bajos o bajos. 
La información producida en nuestro trabajo de campo nos permite caracterizar dos tipos de escenarios que resultan típicos en barrios populares urbanos. Que nos hablan también de otros aglomerados urbanos. Cuando describimos Villa Fiorito, Santa Rosa y San Francisco, estamos describiendo cientos de barrios, donde todo parece similar pero no lo es.

Conceptualizamos entonces, en primer lugar, un primer tipo de escenario barrial que precariza el ejercicio de la salud y los derechos en la adolescencia y juventud. En este tipo de barrios los servicios básicos, las condiciones de vivienda, los niveles educativos y el acceso a instituciones presentan brechas de inequidad con sus respectivos partidos. La vida en las calles de este tipo de escenario barrial es percibida como de baja violencia.

En segundo lugar, conceptualizamos un tipo de escenario crítico, donde además de las brechas en los servicios básicos inexistentes como el agua potable en el hogar o las cloacas, conviven con problemáticas ambientales graves, violencias cotidianas y el estigma que representa vivir en un territorio segregado.

Ahora bien ¿cómo y por qué la especificidad que hemos alcanzado resulta importante para comprender la experiencia de la vulnerabilidad y las desigualdades sociales? A abordar esta pregunta nos dedicaremos en el último apartado.

\section{A MODO DE CIERRE}

En este artículo hemos presentado el proceso metodológico mediante el cual reconstruimos dos escenarios sociales donde transcurren las vidas de adolescentes y jóvenes residentes en barrios populares del Gran Buenos Aires. Los mismos nos hablan de muchos otros barrios, similares a estos, tanto del Gran Buenos Aires como de otros aglomerados urbanos del país.

La noción de escenario barrial nos permite avanzar en la comprensión del análisis de la vulnerabilidad en salud y derechos en la adolescencia y juventud. Por medio de esta noción, es posible documentar cómo las desigualdades sociales se anudan territorialmente y configuran situaciones de vulnerabilidad que afectan la vida de las comunidades.

Como hemos visto a lo largo del artículo, la dimensión territorial permite captar las posiciones estructurales y diferencias de oportunidad constreñidas a un área determinada. En el área del Gran Buenos Aires, el análisis por partido esconde las brechas de desigualdad existentes al interior, mientras que el análisis a nivel barrio aporta especificidad, al describir condiciones heterogéneas en relación a la infraestructura urbana, el acceso a 
recursos y el clima social, las cuales influyen en la salud y los derechos de sus poblaciones.

Tal como lo plantean algunos autores, hemos podido observar a partir de nuestro análisis que el espacio socialmente producido influye no solo en la calidad de la vivienda, de los servicios básicos y del entorno sino sobre todo en las oportunidades y recursos que el territorio viabiliza (o no) como el acceso a la movilidad y el transporte, a la educación, a la salud, al trabajo, entre otros. Asimismo, influye en el estatus simbólico de los habitantes de los barrios segregados (Segura, 2020).

A partir de las cinco dimensiones presentadas hemos llegado a la caracterización de dos tipos de escenarios barriales, los cuales por el nivel de desagregación de los datos utilizados presentan gran especificidad. La potencialidad de esta especificidad muestra intersectadas condiciones de vida, recursos existentes y oportunidades que presenta un área geográfica de baja desagregación. Contar con este tipo de información es de utilidad para comprender las condiciones objetivas de la experiencia de la desigualdad. La información que hemos obtenido en nuestra investigación mediante técnicas cualitativas (utilizando relatos de vida) se potencia en diálogo con la reconstrucción de escenarios barriales. Pues permite poner en relación aquello que sucede en un territorio determinado con la experiencia de los sujetos que lo habitan.

En esta dirección, podremos avanzar en el estudio de cómo se configuran escenarios singulares que afectan la vida de la población joven, y cómo esas condiciones son elementos que debemos ponderar en su especificidad al analizar las trayectorias biográficas de las y los adolescentes y jóvenes que habitan los barrios populares urbanos.

\section{Agradecimientos}

Agradecemos a todo el equipo de estudiantes, becarios/as e investigadores/ as del Proyecto PICT 2346 financiado por el Fondo para la Investigación Científica y Tecnológica de Argentina, en especial a Matilde Schwarz por sus aportes, y a Bianca Gentinetta y Victoria Weisbrot por el procesamiento de datos del Censo Nacional 2010 utilizados para este trabajo. También agradecemos la colaboración de referentes territoriales, docentes y profesionales de la salud que han participado de entrevistas y talleres.

\section{REFERENCIAS BIBLIOGRÁFICAS}

Ayres, José Ricardo; Paiva, Vera y Buchalla, Cassia, 2018, "Derechos humanos y vulnerabilidad en la prevención y promoción de la salud: una introducción”, en 
Vera Paiva, José Ayres, Alejandro Capriati, Mario Pecheny y Ana Amuchastegui (eds). Prevención, Promoción y Cuidado: el enfoque de Vulnerabilidad y Derechos Humanos, TeseoPress, Buenos Aires.

Bourgois, Philippe, 2003, In Search of Respect: Selling Crack in El Barrio. Cambridge University Press, London.

Capriati, A.J., 2014, “'Una aventura abierta': acontecimientos biográficos de jóvenes residentes en villas y barrios populares del Gran Buenos Aires, Argentina”, en Última Década, 22(40), 109-129. Disponible en http://www.scielo.cl/ scielo.php?pid=S0718-22362014000100006\&script=sci_arttext. Consultado el 18/11/2014.

Di Leo, Pablo Francisco y Camarotti, Ana Clara, 2013, Quiero escribir mi historia. Relatos biográficos y procesos de individuación en jóvenes. Biblos, Buenos Aires.

Di Virgilio, Mercedes y Perelman, Mariano, 2014, "Ciudades latinoamericanas. La producción social de las desigualdades urbanas", en M. Di Virgilio y M. Perelman (coords.), Ciudades latinoamericanas. Desigualdad, segregación y tolerancia, Buenos Aires, CLACSO, pp. 9-23.

INDEC, 2014, Necesidades Básicas Insatisfechas (NBI) Información censal del año 2010 (Versión ampliada con datos departamentales). Dirección Nacional de Relaciones Económicas con las Provincias (DINREP), Subsecretaría de Relaciones con Provincias, Ministerio de Economía y Finanzas Públicas de la Nación, 68p. Disponible en http://www2.mecon.gov.ar/hacienda/dinrep/Informes/archivos/NBIAmpliado.pdf. Consultado el 2/11/2017.

INDEC, 2010, Provincia de Buenos Aires, 24 partidos del Gran Buenos Aires. Población total y variación intercensal absoluta y relativa por partido. Años 20012010. Disponible en https://www.indec.gob.ar/ftp/censos/2010/CuadrosDefinitivos/P1-P_Buenos_Aires_24.pdf. Consultado el 3/11/2017.

Kessler, Gabriel y Sabina, Dimarco, 2013, “Jóvenes, policía y estigmatización territorial en la periferia de Buenos Aires", en Espacio Abierto, vol. 22, núm. 2, Universidad del Zulia, Venezuela, 221-243.

Kessler, Gabriel, 2009, El sentimiento de inseguridad. Sociología del temor al delito. Siglo XXI Editores, Buenos Aires. 288 p.

Kornblit, Ana Lía, 2010, Historias y relatos de vida: pseudo análisis y análisis en la investigación social. Ciclo de Seminarios: "Debates metodológicos en proceso de investigación social cualitativa", Universidad de la República, Uruguay; 8 de octubre; Montevideo, Uruguay; p.1-15.

Kornblit, Ana Lía, 2008, Violencia escolar y climas sociales. Buenos Aires: Biblos. 159 p.

Leclerc-Olive, M., 2009, “Temporalidades de la experiencia: las biografías y sus acontecimientos", en Iberofórum. Revista de Ciencias Sociales de la Universidad Iberoamericana, 8, 1-39. 
Pecheny, Mario, 2013, “Desigualdades Estructurales, Salud de Jóvenes LGBT y Lagunas de Conocimiento: ¿Qué Sabemos y qué Preguntamos?”, en Temas em Psicologia, vol. 21, n 2, p.961-972.

Pujadas Muñóz, J., 1992, El método biográfico: El uso de las historias de vida en ciencias sociales. Centro de Investigaciones Sociológicas, Madrid.

Saraví, Gonzalo y Serrano Santos, M. L., 2020, “Jóvenes y territorio: dimensiones espaciales de vulnerabilidad en la transición a la adultez", en Aguilar A. y Escamilla I. (coords.) Expresiones de la segregación residencial y de la pobreza en contexto urbanos y metropolitanos. México, MAPorrúa.

Sawyer, Susan; Azzopardi, Peter; Wickremarathne, Dakshitha; Patton, George, 2018, "The age of adolescence", in Lancet Child Adolesc Health 2 (3): 223-228.

Segura, Ramiro, 2020, “El espacio urbano y la (re)producción de desigualdades sociales. Desacoples entre distribución del ingreso y patrones de urbanización en ciudades latinoamericanas", en Jelin, E; Motta, R. y Costa, S. Repensar las desigualdades. Cómo se producen y entrelazan las asimetrías globales (y qué hace la gente con eso). Buenos Aires, Siglo XXI Editores Argentina.

Sellström, Eva y Bremberg, Sven, 2006, "The significance of neighbourhood context to child andadolescent health and well-being: A systematic review of multilevel studies", in Scandinavian Journal of Public Health 34:5, 544 - 554.

Serrano, M. L., 2019, Bloques de la pared. El proceso de socialización de jóvenes que habitan enclaves de pobreza. Tesis de Doctorado en Antropología Social, CIESAS.

Steinberg, Cora; Tófalo, Ariel; Meschengieser, Carolina y Lotito, Ornella, 2017, "Pensar la relación entre las desigualdades territoriales, sociales y educativas en el ámbito urbano en Argentina: una aproximación cuantitativa y cualitativa" Ponencia presentada en el XIII Congreso Nacional de Ciencia Política La política en entredicho. Volatilidad global, desigualdades persistentes y gobernabilidad democrática, Sociedad Argentina de Análisis Político y la Universidad Torcuato Di Tella, Buenos Aires, 2 al 5 de agosto de 2017.

Steinberg, Cora; Cetrángolo, Oscar y Gatto, Francisco, 2011, Desigualdades territoriales en la Argentina. Insumos para el planeamiento estratégico del sector educativo. Documentos de proyectos CEPAL/UPEA 53. Disponible en https://repositorio.cepal.org/handle/11362/3853. Consultado el 26/04/2018.

UNICEF, 2017, Para cada adolescente una oportunidad. Posicionamiento sobre adolescencia. Buenos Aires: UNICEF Argentina, p. 87. Disponible en https:// www.unicef.org/argentina/spanish/Unicef-Adolescencia-WebFINAL.pdf. Consultado el 23/12/2017.

Wald, Gabriela, 2012, La cultura como recurso. Usos y apropiaciones de dos programas de orquestas juveniles de la Ciudad de Buenos Aires. Tesis doctoral. Doctorado en Ciencias Sociales, Facultad de Ciencias Sociales, Universidad de Buenos Aires, Argentina. 
Zaragaza, Rodrigo y Ronconi, Lucas, 2017, Conurbano infinito. Actores políticos y sociales, entre la presencia estatal y la ilegalidad. Siglo XXI y Fundación Osde, Buenos Aires, 289 p.

\section{RESUMEN CURRICULAR DE LOS AUTORES}

\section{Gabriela Wald}

Es Doctora en Ciencias Sociales (Universidad de Buenos Aires), Master en Educación, Promoción de la Salud y Desarrollo Internacional (Institute of Education, University of London) y Lic. en Ciencias de la Comunicación (Universidad de Buenos Aires). Es investigadora Adjunta del Consejo Nacional de Investigaciones Científicas y Técnicas (CONICET) desde el año 2013, y ha participado y dirigido proyectos de investigación nacionales e internacionales. Se ha desempeñado como consultora en temas de promoción de la salud en infancias y adolescencias de distintos organismos (Unicef Argentina, Ministerio de Salud de la Nación). Sus áreas de trabajo son: vulnerabilidades en la adolescencia y juventud y promoción de la salud comunitaria. Su trabajo se ubica la intersección entre la producción académica y la intervención territorial, con el fin de desarrollar tecnologías de inclusión social a partir de diálogo entre estos saberes.

Dirección electrónica: gawald@gmail.com

Registro ORCID: http://orcid.org/0000-0002-0869-1892

\section{Alejandro Capriati}

Es sociólogo y doctor en ciencias sociales por la Universidad de Buenos Aires (UBA). Se desempeña como Investigador del Consejo Nacional de Investigaciones Científicas y Técnicas (CONICET) y Profesor en la carrera de Sociología en la UBA. Es docente del programa de Doctorado en Ciencias Sociales, Facultad de Ciencias Sociales (UBA), y miembro del Comité Académico de la Maestría en Salud Pública de la Facultad de Medicina, UBA. Ha dirigido y participado en proyectos de investigación en temas de salud, derechos, género y juventud. Publicó Pibes sin calma. Desigualdades y vulnerabilidades en las juventudes argentinas (2019) y compiló Prevención, Promoción y Cuidado: enfoques de Vulnerabilidad y Derechos Humanos (2018, junto a Paiva, Ayres, Pecheny y Amuchastegui). Dirección electrónica: alejandrocapriati@gmail.com Registro ORCID: http://orcid.org/0000-0001-8204-6878 